\title{
The effect of biventricular pacing after coronary artery bypass grafting: A prospective randomized trial of different pacing modes in patients with reduced left ventricular function
}

Frank Eberhardt, MD, ${ }^{\mathrm{a}}$ Matthias Heringlake, MD, ${ }^{\mathrm{b}}$ Maximilian S. Massalme, MD, ${ }^{\mathrm{a}}$ Anika Dyllus, MD, ${ }^{\mathrm{a}}$ Martin Misfeld, MD, PhD, ${ }^{\mathrm{c}}$ Hans-H. Sievers, MD, ${ }^{\mathrm{c}}$ Uwe K. H. Wiegand, MD, ${ }^{\mathrm{a}}$ and Thorsten Hanke, $\mathrm{MD}^{\mathrm{c}}$

Objectives: Biventricular pacing acutely improves left ventricular function in patients with heart failure and left ventricular dyssynchrony. Pressure-volume loop analysis has shown acute perioperative hemodynamic benefits of biventricular pacing immediately after weaning from cardiopulmonary bypass in patients undergoing coronary artery bypass grafting, but whether these effects can be maintained for the early postoperative period is unclear. We hypothesized that biventricular pacing is superior to atrioventricular universal pacing at right ventricular outflowtract and atrial inhibited pacing in patients undergoing coronary artery bypass grafting.

Methods: Ninety-four patients (mean age, $67 \pm 9$ years; mean ejection fraction, 35\% $\pm 4 \%$ ) were prospectively randomized to undergo biventricular, atrioventricular universal, or atrial inhibited pacing at 90 beats/ min for 96 postoperative hours. Clinical end points and postoperative hemodynamics, aminoterminal probrain natriuretic peptide, inotropic support, atrial fibrillation, ventricular arrhythmias, and renal function were evaluated.

Results: Diastolic pulmonary arterial pressure, mean arterial pressure, mixed venous saturation, cardiac index, and cardiac power index did not differ significantly among groups for all time points. Neither raw aminoterminal pro-brain natriuretic peptide nor differential from preoperative values differed significantly among groups at any time point. Median intensive care unit stay (19.5 hours) did not differ significantly by pacing mode. Incidences of postoperative atrial fibrillation were $40 \%$ for atrial inhibited, $29 \%$ for atrioventricular universal, and $37 \%$ for biventricular (differences not significant). Renal function was unaffected by pacing mode.

Conclusion: Despite short-term hemodynamic benefits for patients with reduced left ventricular function, biventricular pacing did not lead to improved postoperative hemodynamics or clinical outcome.

Earn CME credits at

http://cme.ctsnetjournals.org

Atrioventricular synchronous biventricular pacing (DDDBIV) represents an effective therapeutic modality that acutely improves systolic function in the patient with heart failure, left ventricular (LV) ejection fraction (EF) less than $35 \%$, and QRS width greater than $120 \mathrm{~ms}$. $^{1,2}$ The concept of transferring these acute hemodynamic benefits to the cardiac surgical patient with ischemic heart disease and reduced LV function is attractive. Recently, acute improvements in the range of $10 \%$ to $15 \%$ in cardiac output, stroke work, and the first derivative of LV pressure rise have been shown

\footnotetext{
From the Medical Clinic II, ${ }^{\mathrm{a}}$ the Department of Anesthesiology, ${ }^{\mathrm{b}}$ and the Department of Cardiac and Thoracic Vascular Surgery, ${ }^{c}$ University of Lübeck, Lübeck, Germany.

Received for publication July 31, 2008; revisions received Oct 21, 2008; accepted for publication Nov 19, 2008.

Address for reprints: Frank Eberhardt, MD, Universität zu Lübeck, Medizinische Klinik II, Ratzeburger Allee 160, 23538 Lübeck, Germany (E-mail: eberhardt@evkk. de).

J Thorac Cardiovasc Surg 2009;137:1461-7

$0022-5223 / \$ 36.00$

Copyright (c) 2009 by The American Association for Thoracic Surgery

doi:10.1016/j.jtcvs.2008.11.025
}

for DDD-BIV relative to standard atrioventricular synchronous pacing at the right ventricular (RV) outflow tract (DDD-RVOT) in a study of intraoperative pressure-volume loop analysis in patients with ischemic cardiomyopathy after weaning from cardiopulmonary bypass (CPB).

In accordance with these promising intraoperative results, prolonged DDD-BIV might be an additional option after coronary artery bypass grafting (CABG) during the early postoperative period to improve postoperative hemodynamics, reduce postoperative inotrope and vasopressor needs, and improve clinical outcome in patients with reduced LV function. Although the postoperative stability of the additional LV epicardial pacing wire and the general feasibility of DDD-BIV pacing with standard external pacemaker devices have been documented, the overall effect of DDD-BIV relative to standard pacing modes in the early postoperative period is unknown. $^{4,5}$ A prospective, randomized, single-center study was therefore designed to evaluate the effect of biventricular pacing after CABG (http://clinicaltrials.gov/NCT00417092) relative to DDD-RVOT and atrial inhibited pacing (AAI) on early postoperative hemodynamics, arrhythmias, renal function, and clinical outcome parameters in patients with ischemic heart disease and severely reduced LV function ( $\mathrm{EF}<40 \%$ ) undergoing $\mathrm{CABG}$ in a prospective, randomized setting. 


\begin{tabular}{|c|c|}
\hline \multicolumn{2}{|c|}{ Abbreviations and acronyms } \\
\hline AAI & $=$ atrial inhibited pacing \\
\hline CABG & $=$ coronary artery bypass grafting \\
\hline $\mathrm{CPB}$ & $=$ cardiopulmonary bypass \\
\hline DDD-BIV & $\begin{aligned}= & \text { atrioventricular synchronous } \\
& \text { biventricular pacing }\end{aligned}$ \\
\hline \multicolumn{2}{|c|}{$\begin{aligned} \text { DDD-RVOT = } & \text { atrioventricular synchronous pacing } \\
& \text { at right ventricular outflow tract }\end{aligned}$} \\
\hline EF & $=$ ejection fraction \\
\hline GFR & $=$ glomerular filtration rate \\
\hline LV & $=$ left ventricular \\
\hline \multicolumn{2}{|c|}{$\begin{aligned} & \mathrm{NT}-\text { pro-BNP }= \text { aminoterminal pro-brain natriuretic } \\
& \text { peptide }\end{aligned}$} \\
\hline RIFLE & $\begin{array}{l}=\text { risk of renal failure, injury to } \\
\text { kidney, failure of kidney function, } \\
\text { loss of kidney function and } \\
\text { end-stage renal failure }\end{array}$ \\
\hline RV & $=$ right ventricular \\
\hline VVI & $=$ ventricular-only demand pacing \\
\hline
\end{tabular}

\section{MATERIALS AND METHODS Patients}

One hundred patients undergoing elective or urgent CABG with LV EF less than $40 \%$ between December 2005 and June 2007 were included in the study. The study protocol was approved by the institutional review board, and all patients gave written, informed consent.

\section{Protocol}

All patients undergoing CABG, either on a scheduled basis or on an urgent emergency setup, at the University of Lübeck were screened for the study. If EF was less than $40 \%$ at ventriculography, patients were asked to provide written, informed consent to be included in the study. Exclusion criteria were concomitant valve or aortic surgery, presence of a permanent pacemaker or implantable cardioverter defibrillator system, terminal renal failure, and inability to provide written, informed consent. Patients were stratified according to the presence of preoperative paroxysmal or permanent atrial fibrillation (AF) and the presence of preoperative bundle branch block, defined as a QRS width greater than $120 \mathrm{~ms}$. Subsequently, patients received a sealed envelope that contained the random assignment to one of three postoperative pacing modes (DDD-BIV, DDD-RVOT, or AAI). All patients were equipped with a central venous line, an arterial line, and a pulmonary artery thermodilution catheter (Edwards Lifesciences LLC, Irvine, Calif). Fluid management was adjusted to maintain a central venous pressure of 8 to $12 \mathrm{~mm} \mathrm{Hg}$ and a pulmonary arterial diastolic pressure of 15 to $18 \mathrm{~mm} \mathrm{Hg}$. Routine CABG was performed in moderate hypothermia $\left(32^{\circ} \mathrm{C}\right.$ nasopharyngeal temperature) with antegrade blood cardioplegia. Before weaning from CPB, unipolar pacing wires (Streamline 6500; Medtronic, Inc, Minneapolis, Minn) were sutured onto the roof of the right atrium, onto the RV outflow tract in a high paraseptal position, and onto the lateral free wall of the $\mathrm{LV}$ and connected to a dual-chamber external pacemaker (Pace 203H; Osypka, Rheinfelden-Herten, Germany). Weaning from CPB was accomplished in standard fashion.

After weaning from $\mathrm{CPB}$, the randomization envelope was opened, and pacing was conducted as either DDD-BIV, DDD-RVOT, or AAI. Stimulation rate was kept at 90 beats/min for 96 postoperative hours, analogous to the studies on preventing postoperative AF. ${ }^{6}$ Patients with sustained AF after weaning from $\mathrm{CPB}$ were excluded from further analysis. If AF occurred during the further postoperative period, the external pacemaker was programmed to ventricular-only demand pacing (VVI) for the duration of AF and switched back to the randomized pacing mode after termination of the AF episode. VVI pacing during AF was instituted along the same principles as the randomized pacing modes as minimal ventricular pacing (AAI 90 beats/min changed to VVI at 50 beats/min), RV outflow tract pacing (DDD-RVOT at 90 beats/min changed to RV outflow tract VVI at 90 beats/min), or biventricular pacing (DDD-BIV at 90 beats/min changed to biventricular VVI at 90 beats/min). Termination of AF was attempted either by intravenous infusion of amiodarone $(450 \mathrm{mg}$ ) during 2 hours followed by constant amiodarone infusion $(900 \mathrm{mg} / \mathrm{d})$ or by immediate electrical cardioversion in a standard fashion in case of hemodynamic instability. Postoperative $\beta$-blocker therapy was instituted if hemodynamically tolerable. The atrioventricular delay was programmed at $150 \mathrm{~ms}$ during DDD-RVOT and $120 \mathrm{~ms}$ during DDD-BIV to ensure true biventricular capture. Atrial sensitivity was programmed at a default value of $0.5 \mathrm{mV}$ and could be lowered to $0.2 \mathrm{mV}$ to obtain at least a 3 -fold safety margin. Ventricular sensitivity was programmed to a minimum programmable value of $1.0 \mathrm{mV}$. Pacing output was programmed to a 3 -fold safety margin. Lower safety margins were allowed in case of chest wall stimulation. Pacing and sensing thresholds were measured every 8 hours to ensure proper pacemaker function. After surgery, the patient was transferred to the intensive care unit. In accordance with the institutional standard, patients were transferred to an intermediate care unit within 24 hours if in clinically stable condition without the need for catecholamines or inotropes.

\section{Hemodynamic Parameters and Inotropes}

If the heart was clinically hypocontractile or the stroke volume index was below $35 \mathrm{~mL} / \mathrm{m}^{2}$ despite raised filling pressures (central venous pressure $>15 \mathrm{~mm} \mathrm{Hg}$, pulmonary arterial diastolic pressure $>18 \mathrm{~mm} \mathrm{Hg}$ ), inotropic support with enoximone (INN) and dobutamine was started. ${ }^{7}$ Norepinephrine was added to maintain mean arterial pressure above $65 \mathrm{~mm} \mathrm{Hg}$. Cumulative and maximum doses of enoximone, dobutamine, and norepinephrine were recorded for each patient.

Central venous pressure, mean and diastolic pulmonary arterial pressures, mean arterial pressure, and mixed venous saturation were measured hourly until removal of the thermodilution catheter. Thermodilution cardiac output, cardiac index, was determined hourly as the average of three measurements. Cardiac power index was calculated to correct cardiac index for ventricular afterload as follows ${ }^{8}$ : cardiac power index $=$ cardiac index/mean arterial pressure $\times 0.00222 \mathrm{~W} / \mathrm{s}$.

\section{Arrhythmias}

Patients were continuously monitored (Infinity Kappa; Dräger, Lübeck, Germany). The numbers of AF episodes, ventricular tachycardia episodes, and defibrillations were noted, as was the cumulative amiodarone dose.

\section{Serum Parameters}

Creatine kinase MB fraction and troponin $\mathrm{T}$ were measured preoperatively, immediately postoperatively on arrival at the intensive care unit, and at 24 and 96 postoperative hours. For the determination of the aminoterminal pro-brain natriuretic peptide (NT-pro-BNP), ethylenediaminetetraacetic acid-treated blood samples were also collected at these time points, immediately centrifuged at $3500 \mathrm{~g}$ for 10 minutes, and stored at $-70^{\circ} \mathrm{C}$. NT-pro-BNP was measured with commercially available assays (ECLIA Elecsys 2010 analyzer; F. Hoffmann-La Roche AG, Basel, Switzerland) in plasma samples never previously thawed.

\section{Renal Function}

Urinary output was measured hourly. Creatinine concentrations were measured every 8 hours during the first 24 hours and every 24 hours thereafter. Glomerular filtration rate (GFR) was determined by the Modification of Diet in Renal Disease formula. GFR and urinary output were combined to 
TABLE 1. Preoperative patient data and medications

\begin{tabular}{|c|c|c|c|c|}
\hline & All $(n=94)$ & $\mathbf{A A I}(\mathbf{n}=\mathbf{3 0})$ & DDD-RVOT $(\mathbf{n}=31)$ & DDD-BIV $(\mathbf{n}=\mathbf{3 3})$ \\
\hline Age $(y$, mean \pm SD) & $67 \pm 9$ & $66 \pm 10$ & $67 \pm 9$ & $67 \pm 7$ \\
\hline Sex $(\%$ male $)$ & $77 \%$ & $84 \%$ & $70 \%$ & $75 \%$ \\
\hline Euroscore $($ mean $\pm \mathrm{SD})$ & $9.2 \pm 2.7$ & $8.9 \pm 2.7$ & $9.3 \pm 2.8$ & $8.0 \pm 2.6$ \\
\hline New York Heart Association functional class (mean \pm SD) & $2.1 \pm 1.2$ & $2.1 \pm 1.1$ & $1.9 \pm 1.3$ & $2.2 \pm 1.2$ \\
\hline Canadian Cardiovascular Society class (mean $\pm \mathrm{SD}$ ) & $2.0 \pm 1.5$ & $2.0 \pm 1.6$ & $1.7 \pm 1.3$ & $2.2 \pm 1.7$ \\
\hline Mitral regurgitation (mean $\pm \mathrm{SD}$ ) & $1.0 \pm 0.8$ & $1.1 \pm 0.9$ & $0.8 \pm 0.8$ & $1.1 \pm 0.8$ \\
\hline Previous coronary artery bypass grafting $(\%)$ & $3 \%$ & $7 \%$ & $6 \%$ & $0 \%$ \\
\hline Previous myocardial infarction $(\%)$ & $60 \%$ & $73 \%$ & $53 \%$ & $58 \%$ \\
\hline Acute myocardial infarction $(\%)$ & $20 \%$ & $13 \%$ & $29 \%$ & $16 \%$ \\
\hline Ejection fraction $(\%$, mean $\pm \mathrm{SD})$ & $35 \% \pm 4 \%$ & $35 \% \pm 3 \%$ & $34 \% \pm 5 \%$ & $34 \% \pm 5 \%$ \\
\hline Bundle-branch block (\%) & $6 \%$ & $7 \%$ & $0 \%$ & $10 \%$ \\
\hline QRS width $(\mathrm{ms}$, mean $\pm \mathrm{SD})$ & $100 \pm 16$ & $98 \pm 16$ & $102 \pm 12$ & $99 \pm 20$ \\
\hline $\mathrm{PQ}$ time $(\mathrm{ms}$, mean $\pm \mathrm{SD})$ & $165 \pm 29$ & $163 \pm 32$ & $163 \pm 25$ & $168 \pm 32$ \\
\hline Preoperative sinus rhythm (\%) & $88 \%$ & $93 \%$ & $88 \%$ & $84 \%$ \\
\hline Troponin T positivity (\%) & $29 \%$ & $40 \%$ & $24 \%$ & $26 \%$ \\
\hline \multicolumn{5}{|l|}{ Preoperative medication $(\%)$} \\
\hline Aspirin & $70 \%$ & $80 \%$ & $76 \%$ & $56 \%$ \\
\hline Clopidogrel & $16 \%$ & $20 \%$ & $18 \%$ & $11 \%$ \\
\hline$\beta$-Blocker & $88 \%$ & $73 \%$ & $94 \%$ & $94 \%$ \\
\hline Calcium-channel antagonist & $26 \%$ & $40 \%$ & 24 & 17 \\
\hline Angiotensin-converting enzyme inhibitor & $68 \%$ & $73 \%$ & 71 & 61 \\
\hline Angiotensin II type 1 antagonist & $18 \%$ & $20 \%$ & $6 \%$ & $22 \%$ \\
\hline Aldosterone antagonist & $15 \%$ & $17 \%$ & $10 \%$ & $18 \%$ \\
\hline Loop diuretic & $46 \%$ & $40 \%$ & $41 \%$ & $56 \%$ \\
\hline Thiazide & $23 \%$ & $20 \%$ & $23 \%$ & $27 \%$ \\
\hline Statin & $82 \%$ & $79 \%$ & $89 \%$ & $76 \%$ \\
\hline
\end{tabular}

There were no significant differences among the groups. $A A I$, Atrial inhibited pacing; $D D D-R V O T$, atrioventricular synchronous pacing at right ventricular outflow tract; $D D D-B I V$, atrioventricular synchronous biventricular pacing.

obtain the postoperative RIFLE (risk of renal failure, injury to kidney, failure of kidney function, loss of kidney function, and end-stage renal failure) score. ${ }^{9}$ Briefly, a RIFLE score of 1 (risk) is defined as a decrease in GRF greater than $25 \%$ or a urinary output less than $0.5 \mathrm{~mL} /(\mathrm{kg} \cdot \mathrm{h})$ for 6 hours, a RIFLE score of 2 (injury) is defined as a decrease in GFR greater than $50 \%$ or a urinary output less than $0.5 \mathrm{~mL} /(\mathrm{kg} \cdot \mathrm{h})$ for 12 hours, and a RIFLE score of 3 (failure) is defined as a decrease in GFR greater than $75 \%$ or a urinary output less than $0.3 \mathrm{~mL} /(\mathrm{kg} \cdot \mathrm{h})$ for 24 hours or anuria. Cumulative RIFLE scores for the entire postoperative period were obtained for each patient. Moreover, the use of continuous venovenous hemofiltration was noted.

\section{Clinical Outcome Parameters}

The 28-day survival status, the intensive care unit stay, and the hospital stay were recorded for each patient. Duration of intensive care unit stay was left to the clinical discretion of the intensive care unit physician in charge. Further clinical outcome parameters were postoperative myocardial infarction, need for postoperative revascularization, use of an intra-aortic balloon pump, rethoracotomy, and stroke.

\section{Statistical Analysis}

Statistical analyses were performed with SPSS for Windows version 10.0.1 (SPSS Inc, Chicago, Ill). Data are expressed as mean \pm SD. Results per treatment group were analyzed on an intent-to-treat basis.

Continuous variables were analyzed for differences between pacing groups by 1-way analysis of variance with post hoc Bonferroni correction with a $95 \%$ confidence interval. Categoric variables were compared with the $\chi^{2}$ test. On the basis of hemodynamic data obtained by pressure-volume loop analysis, ${ }^{3}$ the trial was designed to detect a $15 \%$ difference in cardiac power index assuming an average a priori value of $0.6 \pm 0.10 \mathrm{~W} / \mathrm{m}^{2}$ at an $\alpha$ value of .05 and a statistical power of .80 . Under the design assumptions, a target size of 33 patients per group was calculated.

\section{RESULTS}

\section{Preoperative and Intraoperative Data}

Follow-up was $100 \%$ complete. Six patients were excluded from the analysis because of incomplete study data

TABLE 2. Intraoperative data

\begin{tabular}{|c|c|c|c|c|}
\hline & All $(n=94)$ & $\mathbf{A A I}(\mathbf{n}=\mathbf{3 0})$ & DDD-RVOT $(\mathrm{n}=31)$ & DDD-BIV $(\mathbf{n}=\mathbf{3 3})$ \\
\hline Aortic crossclamp time (min, mean $\pm \mathrm{SD}$ ) & $86 \pm 37$ & $81 \pm 32$ & $88 \pm 43$ & $87 \pm 34$ \\
\hline Cardiopulmonary bypass time (min, mean $\pm \mathrm{SD}$ ) & $105 \pm 39$ & $106 \pm 36$ & $100 \pm 28$ & $115 \pm 42$ \\
\hline Veins (No., mean $\pm \mathrm{SD}$ ) & $2.0 \pm 1.1$ & $1.9 \pm 1.1$ & $2.2 \pm 1.0$ & $1.8 \pm 1.2$ \\
\hline Left internal thoracic artery graft $(\%)$ & $93 \%$ & $90 \%$ & $94 \%$ & $94 \%$ \\
\hline Second arterial bypass $(\%)$ & $19 \%$ & $23 \%$ & $6 \%$ & $27 \%$ \\
\hline Intra-aortic balloon pump $(\%)$ & $4.3 \%$ & $0 \%$ & $1.1 \%$ & $3.2 \%$ \\
\hline
\end{tabular}

There were no significant differences among the groups. $A A I$, Atrial inhibited pacing; $D D D-R V O T$, atrioventricular synchronous pacing at right ventricular outflow tract; $D D D-B I V$, atrioventricular synchronous biventricular pacing. 
TABLE 3. Clinical end points in relation to pacing mode

\begin{tabular}{|c|c|c|c|c|}
\hline & $\begin{array}{c}\text { All } \\
(n=94)\end{array}$ & $\begin{array}{c}\text { AAI } \\
(\mathbf{n}=\mathbf{3 0}) \\
\end{array}$ & $\begin{array}{c}\text { DDD-RVOT } \\
(\mathbf{n}=\mathbf{3 1})\end{array}$ & $\begin{array}{c}\text { DDD-BIV } \\
(\mathbf{n}=\mathbf{3 3}) \\
\end{array}$ \\
\hline Mortality (No.) & 3 & 1 & 1 & 1 \\
\hline $\begin{array}{l}\text { Cerebrovascular } \\
\text { accident (No.) }\end{array}$ & 1 & 0 & 1 & 0 \\
\hline $\begin{array}{l}\text { Postoperative } \\
\text { myocardial infarction } \\
\text { (No.) }\end{array}$ & 1 & 0 & 0 & 0 \\
\hline $\begin{array}{l}\text { Postoperative } \\
\text { revascularization (No.) }\end{array}$ & 1 & 0 & 0 & 1 \\
\hline $\begin{array}{l}\text { Intra-aortic balloon } \\
\text { pump (No.) }\end{array}$ & 4 & 0 & 1 & 3 \\
\hline $\begin{array}{c}\text { Continuous venovenous } \\
\text { hemofiltration (No.) }\end{array}$ & 3 & 0 & 1 & 2 \\
\hline Rethoracotomy (No.) & 4 & 1 & 0 & 3 \\
\hline \multicolumn{5}{|l|}{$\begin{array}{l}\text { Intensive care } \\
\text { unit stay (h) }\end{array}$} \\
\hline Mean \pm SD & $52 \pm 93$ & $44 \pm 93$ & $37 \pm 51$ & $74 \pm 122$ \\
\hline Median & 20 & 21 & 19 & 20 \\
\hline $\begin{array}{l}\text { Combined end point } \\
\text { without } \\
\text { rethoracotomy (No.) }\end{array}$ & 10 & 1 & 3 & 6 \\
\hline
\end{tabular}

There were no significant differences among the groups. $A A I$, Atrial inhibited pacing; $D D D-R V O T$, atrioventricular synchronous pacing at right ventricular outflow tract; $D D D-B I V$, atrioventricular synchronous biventricular pacing.

$(n=4)$ or withdrawal of informed consent $(n=2)$. Preoperative patient characteristics and preoperative medication were consistent with a patient population with ischemic cardiomyopathy at an increased perioperative risk with a mean
EuroSCORE of 9.2 (Table 1). Intraoperative patient characteristics revealed no significant differences among pacing groups (Table 2).

\section{Outcome Data}

Clinical outcome parameters are presented in Table 3. No deaths were recorded during the stimulation period of 96 hours. Further early (30-day) mortality was 3.1\% $(n=3)$. Causes of death were cardiac in 1 case (cardiogenic shock) and noncardiac in 2 (septic shock). The overall incidences of relevant clinical end points were comparatively low (Table 3 ). The randomly assigned pacing mode could be maintained for 96 hours in $96 \%$ of the AAI group, 94\% of the DDD-RVOT group, and $92 \%$ of the DDD-BIV group. Reason for pacing discontinuation was lead failure with loss of stimulation or sensing that could not be rectified by adjustment of the temporary pacemaker device. Pacing had to be discontinued in 1 patient in the DDD-BIV group because of diaphragmatic stimulation. With the exception of central venous pressure, no significant differences could be detected among groups for any hemodynamic parameter (Figure 1). Mean cardiac index at 12 postoperative hours was $3.23 \pm$ $0.74 \mathrm{~L} /\left(\min \cdot \mathrm{m}^{2}\right)$; mean cardiac power index was $0.54 \pm$ $0.14 \mathrm{~W} / \mathrm{m}^{2}$. The median preoperative NT-pro-BNP level was $1669 \mathrm{pg} / \mathrm{mL}$ (Figure 2). NT-pro-BNP, troponin T, and creatine kinase levels were in the same range at various preoperative and postoperative time points, with no significant differences among pacing groups. Differential values between preoperative and postoperative measurements did
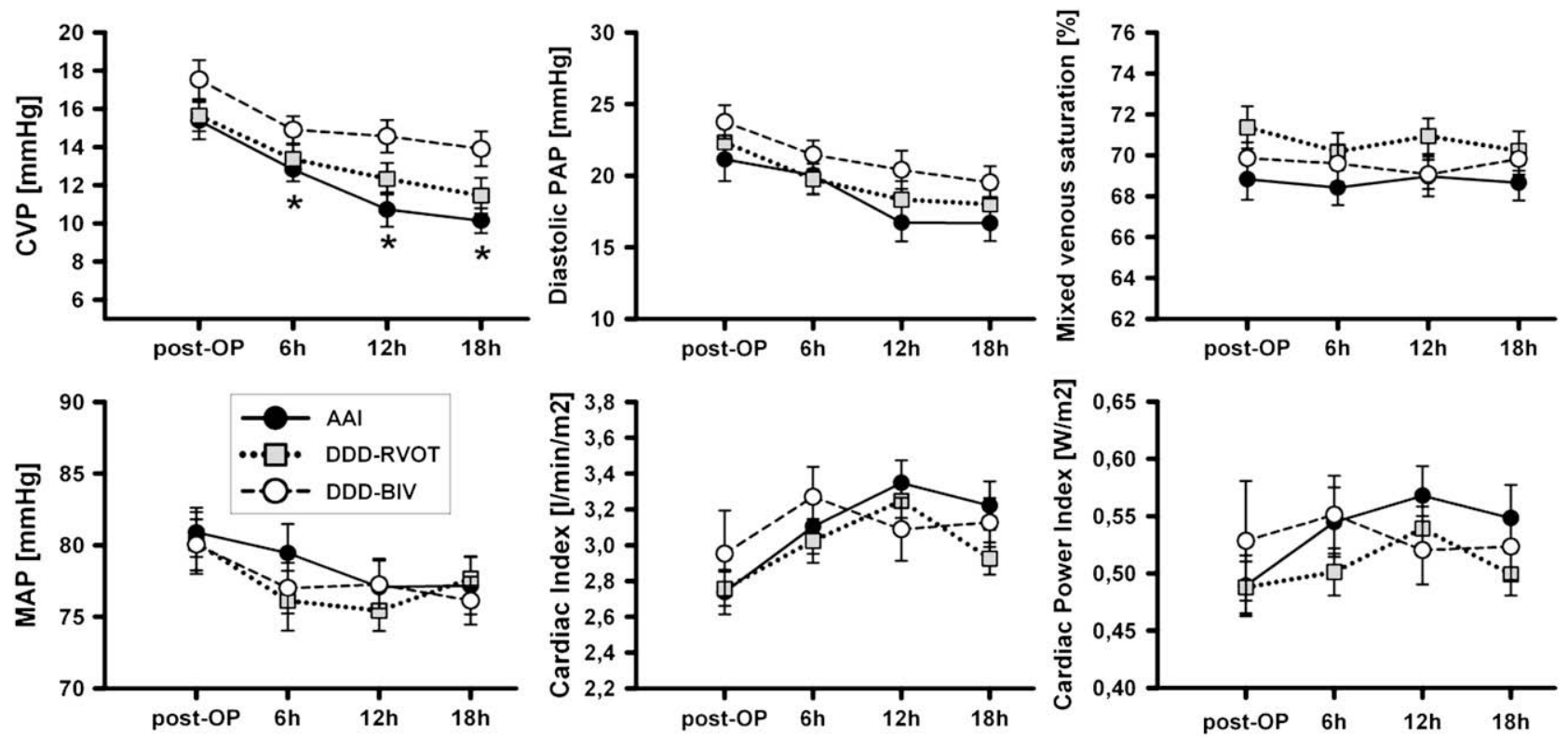

FIGURE 1. Postoperative hemodynamics in relation to postoperative pacing mode. Significant differences between atrial inhibited pacing and atrioventricular synchronous biventricular pacing $(P<.05)$ are marked with asterisks. $C V P$, Central venous pressure; $P A P$, pulmonary arterial pressure; $M A P$, mean arterial pressure; $A A I$, atrial inhibited pacing; $D D D-R V O T$, atrioventricular synchronous pacing at right ventricular outflow tract; $D D D-B I V$, atrioventricular synchronous biventricular pacing. 

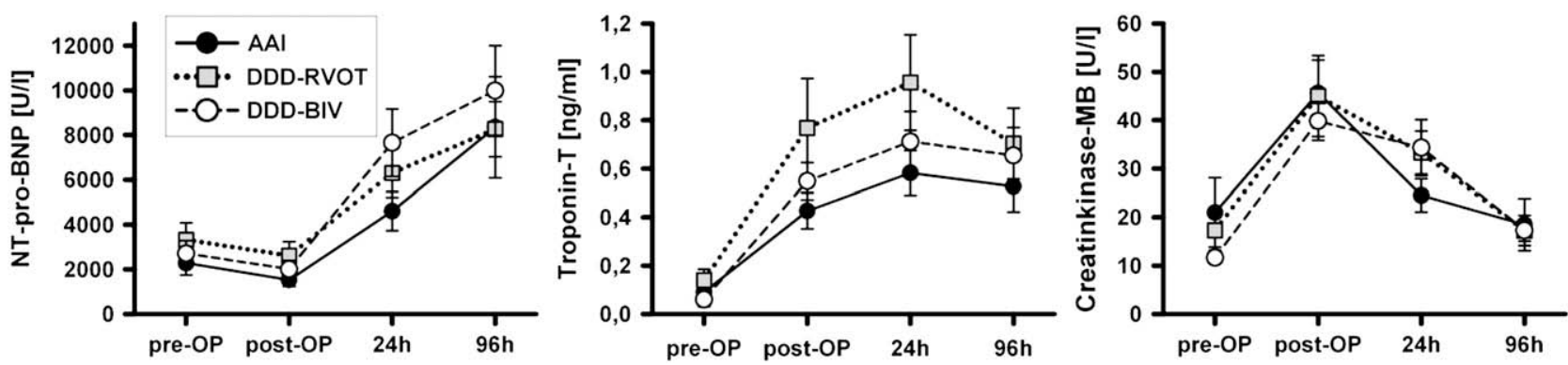

FIGURE 2. Time courses of aminoterminal pro-brain natriuretic peptide (NT-pro-BNP, left panel), troponin T (middle panel), and creatine kinase isoenzyme $\mathrm{MB}(C K-M B$, right panel) in relation to pacing mode. There were no significant differences among pacing groups. AAI, Atrial inhibited pacing; $D D D-R V O T$, atrioventricular synchronous pacing at right ventricular outflow tract; $D D D-B I V$, atrioventricular synchronous biventricular pacing; pre-OP, preoperative; post-OP, postoperative.

not yield significant differences among groups. Postoperative application of inotropes and vasopressors did not differ among pacing groups (Figure 3). The incidences of $\mathrm{AF}$ and ventricular arrhythmias and the use of amiodarone are displayed in Figure 4. Although the incidence of AF was similar among groups, patients in the DDD-BIV group did show a tendency toward higher amiodarone doses than patients in the AAI group to maintain sinus rhythm $(P=.05)$. Preoperative creatinine clearance was normal and did not change significantly in the postoperative time course (Figure 5). RIFLE score was significantly lower only at 24 postoperative hours in the DDD-RVOT group relative to the DDD-BIV group. The number of patients with a cumulative RIFLE score greater than 1 was not statistically different among groups for the entire observational period (AAI 17\%, DDD-RVOT 16\%, DDD-BIV 26\%).

\section{DISCUSSION}

To our knowledge, this is the first prospective, randomized study to compare in a 96-hour period temporary postoperative DDD-BIV with standard pacing modes in a homogeneous population of patients with depressed LV function undergoing pure coronary artery revascularization. No significant differences were seen among DDD-BIV, DDD-RVOT, and AAI groups for a variety of clinical and hemodynamic parameters.

\section{DDD-BIV After CABG}

DDD-BIV improves symptoms, quality of life, exercise tolerance, and mortality for patients with acute and chronic heart failure who have an EF less than $35 \%$ and a widened QRS complex. ${ }^{10,11}$ An acute response to DDD-BIV among these patients is documented during pressure-volume loop analysis, and these effects are maintained for at least 6 months. ${ }^{1,2,12}$ DDD-BIV to improve LV function after general cardiac surgery has been investigated in a few studies, with diverse results. ${ }^{5,13-16}$ Several methodologic drawbacks apply to these studies. Some studies included patients with additional valve surgery ${ }^{5,15}$ or patients with maintained LV function. ${ }^{13}$ Other studies used paraseptal pacing sites for both RV and LV leads, although the free lateral wall has proved to be the most effective LV pacing wire position. ${ }^{17}$ All studies have in common that only a single, sometimes variable point in time was used for hemodynamic measurements after cardiac surgery. A pressure-volume loop analysis immediately after weaning from $\mathrm{CPB}$ in patients with normal QRS complex and severely reduced LV function $(\mathrm{EF}<35 \%)$ showed improvement in systolic function parameters in the range of $10 \%$ to $15 \%$ for DDD-BIV and AAI relative to DDD-RVOT pacing, with diastolic parameters remaining largely unaffected. ${ }^{3}$ These acute hemodynamic effects, however, only apply to the immediate perioperative period after weaning from $\mathrm{CPB}$ in
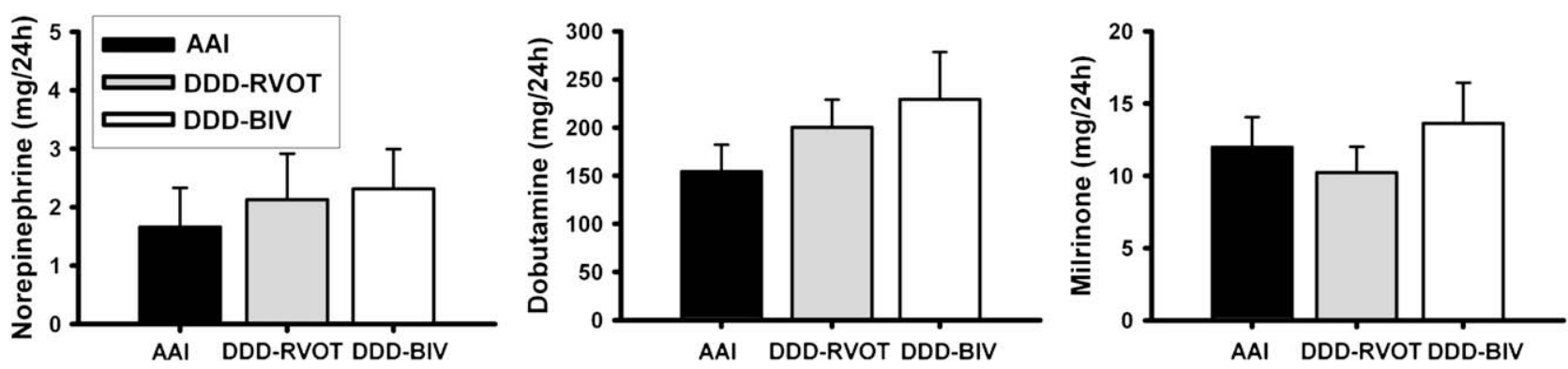

FIGURE 3. Cumulative doses during first 24 postoperative hours for norepinephrine (noradrenaline, left panel), dobutamine (middle panel), and milrinone (right panel) in relation to pacing mode. There were no significant differences among pacing groups. AAI, Atrial inhibited pacing; DDD-RVOT, atrioventricular synchronous pacing at right ventricular outflow tract; $D D D-B I V$, atrioventricular synchronous biventricular pacing. 

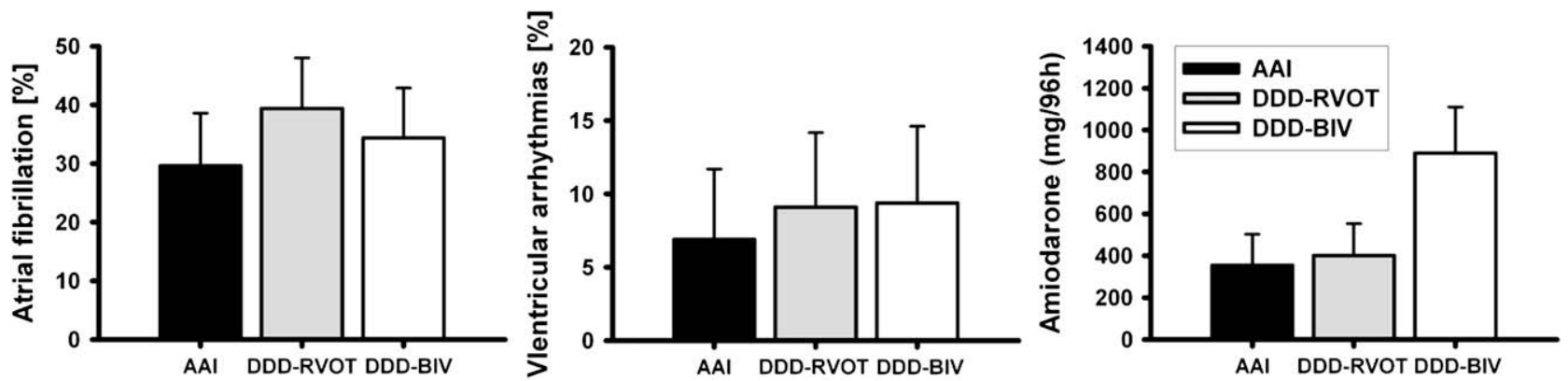

FIGURE 4. Cumulative incidences of atrial fibrillation (left panel) and ventricular arrhythmias (sustained ventricular tachycardia and ventricular fibrillation, middle panel) and amiodarone dose (right panel) during 96-hour stimulation period in relation to pacing mode. There were no significant differences among pacing groups. $A A I$, Atrial inhibited pacing; $D D D-R V O T$, atrioventricular synchronous pacing at right ventricular outflow tract; $D D D-B I V$, atrioventricular synchronous biventricular pacing.

an open-chest situation with rapid volume changes and high levels of inotropes or vasopressors.

This study was designed to determine from longitudinal measurements whether these beneficial hemodynamic effects of DDD-BVI and AAI relative to DDD-RVOT could be maintained for the early 96-hour postoperative period. Additional positive hemodynamic or clinical effects of DDD-BIV relative to DDD-RVOT and AAI were not detectable during the study period. Cardiac power index, which proved to be the strongest hemodynamic correlate of mortality during cardiogenic shock in the SHOCK trial, ${ }^{8}$ did not differ among stimulation groups. Mixed venous saturation, which has been proved to be a valid prognostic factor in the postoperative care of cardiac surgical patients, ${ }^{18}$ was also similar among pacing groups. Levels of enzymes relevant to cardiac surgery (eg, NT-pro-BNP, creatine kinase, $\mathrm{MB}$ fraction of creatine kinase) were in the typical range for a patient population with severely reduced LV function preoperatively and did not differ among groups postoperatively. ${ }^{19-21}$ The hemodynamic results are supported by the inotrope and vasopressor needs, which were not significantly different among groups. Additionally, the overall incidence of renal dysfunction was low in our patient population, with no overall differences among groups at 96 post- operative hours. The average incidence of postoperative AF was in the same range as reported previously for patients with reduced $\mathrm{LV}$ function undergoing $\mathrm{CABG}^{6}$ and was not influenced by the choice of pacing mode.

Essentially, DDD-BIV in our study was not superior to DDD-RVOT and AAI with regard to a wide variety of parameters in the observed patient group and even showed a tendency toward a negative outcome for some parameters. Although the distributions of preoperative and intraoperative characteristics were similar among stimulation groups, 3 patients in the DDD-BIV group had prolonged intensive care unit stays, with concurrent adverse hemodynamics and vasopressor and inotrope needs because of respirator-associated pneumonia and postoperative sepsis. This may have tilted the results in an unfavorable direction for DDD-BIV. Another factor that might explain the lack of superiority of DDD-BIV is that the observed patient population was not preoperatively screened for mechanical dyssynchrony. Mechanical dyssynchrony can be determined by QRS width or echocardiographic parameters; however, the relationship between QRS duration and LV intraventricular dyssynchrony is not very strong, and a high degree of dyssynchrony may be present in patients with normal QRS complexes. ${ }^{22}$ Moreover, the relationship between preoperative and
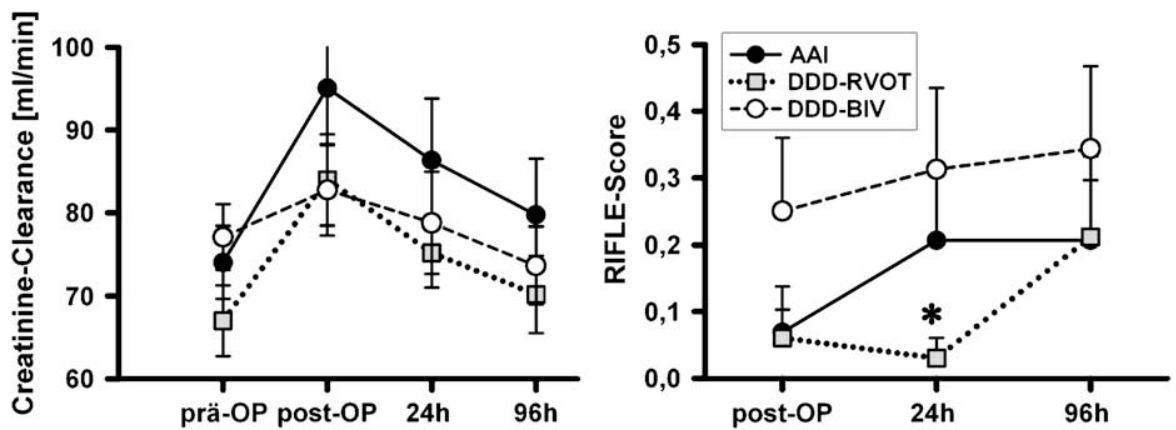

FIGURE 5. Creatinine clearance (by Modification of Diet in Renal Disease method, left panel) and RIFLE score (left panel) in relation to pacing mode. Significant difference $(P<.05)$ between atrioventricular synchronous pacing at right ventricular outflow tract and atrioventricular synchronous biventricular pacing is marked with asterisk. AAI, Atrial inhibited pacing; $D D D-R V O T$, atrioventricular synchronous pacing at right ventricular outflow tract; $D D D-B I V$, atrioventricular synchronous biventricular pacing; pre-OP, preoperative; post-OP, postoperative. 
postoperative dyssynchrony after CPB is unclear. Mechanical dyssynchrony occurs after weaning from $\mathrm{CPB}$ as a result of regional myocardial edema despite normal preoperative QRS complexes. ${ }^{23}$ In this situation, both DDD-BIV and AAI have been reported to be hemodynamically superior to DDD-RVOT in a similar patient population immediately after weaning from $\mathrm{CPB} .{ }^{3}$ With restoration of myocardial function after cardiac surgery with the use of extracorporeal circulation, the positive effect of DDD-BIV obviously disappears. On the basis of the observed data, DDD-BIV thus lacks clinical and hemodynamic superiority in the early postoperative period for patients with severely reduced LV function and normal QRS complex. Even if the potentially deleterious effects of RV pacing are to be avoided in those patients who require mandatory pacing for perioperative bradyarrythmia, DDD-BIV is not superior to DDDRVOT. $^{24,25}$ This finding may also be explained by the fact that epicardial high paraseptal DDD-RVOT results in less LV dyssynchrony than does endocardial apical RV pacing. ${ }^{24}$ Although it remains a matter of speculation, postoperative DDD-BIV pacing might be of benefit for a carefully selected cardiac surgical subgroup of patients with severely reduced LV function (EF $\leq 25 \%$ ), a wide QRS complex, or documented interventricular dyssynchrony.

\section{LIMITATIONS}

Variations of all three pacing wire positions are possible. Although left atrial or biatrial pacing has been suggested to reduce the incidence of postoperative $\mathrm{AF}$, conclusive evidence of clinical superiority relative to right atrial pacing is lacking. ${ }^{6}$ In general, the outcome after cardiac surgery in a high-risk patient population is governed by many clinical factors, some of which are difficult to control for in a prospective, randomized study design.

\section{CONCLUSIONS}

Routine use of temporary DDD-BIV pacing in patients undergoing $\mathrm{CABG}$ with severely reduced LV function and normal QRS complex cannot be recommended on the basis of the observed data. DDD-BIV pacing in this patient cohort did not improve postoperative hemodynamics or clinical outcome relative to DDD-RVOT or AAI.

\section{References}

1. Kass DA, Chen CH, Curry C, Talbot M, Berger R, Fetics B, et al. Improved left ventricular mechanics from acute VDD pacing in patients with dilated cardiomyopathy and ventricular conduction delay. Circulation. 1999;99:1567-73.

2. Blanc JJ, Etienne Y, Gilard M, Mansourati J, Munier S, Boschat J, et al. Evaluation of different ventricular pacing sites in patients with severe heart failure: results of an acute hemodynamic study. Circulation. 1997;96:3273-7.

3. Eberhardt F, Hanke T, Bechtel M, Bonnemeier H, Wiegand UK, Misfeld M. Evaluation of different pacing modes after coronary artery bypass grafting using pressure volume loops. Heart Rhythm. 2006;3:S23-4.

4. Eberhardt F, Hanke T, Heringlake M, Massalme MS, Bechtel M, Misfeld M, et al. Feasibility of temporary biventricular pacing in patients with reduced left ventric- ular function after coronary artery bypass grafting. Pacing Clin Electrophysiol. 2007;30(Suppl. 1):S50-3.

5. Dzemali O, Bakhtiary F, Dogan S, Wittlinger T, Moritz A, Kleine P. Perioperative biventricular pacing leads to improvement of hemodynamics in patients with reduced left-ventricular function-interim results. Pacing Clin Electrophysiol. 2006;29:1341-5.

6. Crystal E, Connolly SJ, Sleik K, Ginger TJ, Yusuf S. Interventions on prevention of postoperative atrial fibrillation in patients undergoing heart surgery: a metaanalysis. Circulation. 2002;106:75-80.

7. Carl M, Alms A, Braun J, Dongas A, Erb J, Goetz A, et al. Guidelines for intensive care in cardiac surgery patients: haemodynamic monitoring and cardio-circulatory treatment guidelines of the German Society for Thoracic and Cardiovascular Surgery and the German Society of Anaesthesiology and Intensive Care Medicine. Thorac Cardiovasc Surg. 2007;55:130-48.

8. Fincke R, Hochman JS, Lowe AM, Menon V, Slater JN, Webb JG, et al. Cardiac power is the strongest hemodynamic correlate of mortality in cardiogenic shock: a report from the SHOCK trial registry. J Am Coll Cardiol. 2004;44:340-8.

9. Bellomo R, Kellum JA, Ronco C. Defining and classifying acute renal failure: from advocacy to consensus and validation of the RIFLE criteria. Intensive Care Med. 2007;33:409-13.

10. Abraham WT, Fisher WG, Smith AL, Delurgio DB, Leon AR, Loh E, et al. Cardiac resynchronization in chronic heart failure. $N$ Engl J Med. 2002;346:1845-53.

11. Bristow MR, Saxon LA, Boehmer J, Krueger S, Kass DA, De Marco T, et al. Cardiac-resynchronization therapy with or without an implantable defibrillator in advanced chronic heart failure. N Engl J Med. 2004;350:2140-50.

12. Steendijk P, Tulner SA, Bax JJ, Oemrawsingh PV, Bleeker GB, van Erven L, et al Hemodynamic effects of long-term cardiac resynchronization therapy: analysis by pressure-volume loops. Circulation. 2006;113:1295-304.

13. Foster AH, Gold MR, McLaughlin JS. Acute hemodynamic effects of atrio-biventricular pacing in humans. Ann Thorac Surg. 1995;59:294-300.

14. Weisse U, Isgro F, Werling C, Lehmann A, Saggau W. Impact of atrio-biventricular pacing to poor left-ventricular function after CABG. Thorac Cardiovasc Surg. 2002;50:131-5

15. Saxon LA, Kerwin WF, Cahalan MK, Kalman JM, Olgin JE, Foster E, et al. Acute effects of intraoperative multisite ventricular pacing on left ventricular function and activation/contraction sequence in patients with depressed ventricular function. J Cardiovasc Electrophysiol. 1998;9:13-21.

16. Schmidt C, Frielingsdorf J, Debrunner M, Tavakoli R, Genoni M, Straumann E et al. Acute biventricular pacing after cardiac surgery has no influence on regional and global left ventricular systolic function. Europace. 2007;9:432-6.

17. Butter C, Auricchio A, Stellbrink C, Fleck E, Ding J, Yu Y, et al. Effect of resynchronization therapy stimulation site on the systolic function of heart failure patients. Circulation. 2001;104:3026-9.

18. Hausmann H, Potapov EV, Koster A, Krabatsch T, Stein J, Yeter R, et al. Prognosis after the implantation of an intra-aortic balloon pump in cardiac surgery calculated with a new score. Circulation. 2002;106(12 Suppl.1):I203-6.

19. Hartmann F, Packer M, Coats AJ, Fowler MB, Krum H, Mohacsi P, et al Prognostic impact of plasma N-terminal pro-brain natriuretic peptide in severe chronic congestive heart failure: a substudy of the Carvedilol Prospective Randomized Cumulative Survival (COPERNICUS) trial. Circulation. 2004;110: 1780-6.

20. Jogia PM, Kalkoff M, Sleigh JW, Bertinelli A, La Pine M, Richards AM, et al NT-pro BNP secretion and clinical endpoints in cardiac surgery intensive care patients. Anaesth Intensive Care. 2007;35:363-9.

21. Brown JR, Hernandez F Jr, Klemperer JD, Clough RA, DiPierro FV, Hofmaster PA, et al. Cardiac troponin T levels in on- and off-pump coronary artery bypass surgery. Heart Surg Forum. 2007;10:E42-6.

22. Achilli A, Sassara M, Ficili S, Pontillo D, Achilli P, Alessi C, et al. Long-term ef fectiveness of cardiac resynchronization therapy in patients with refractory heart failure and "narrow" QRS. J Am Coll Cardiol. 2003;42:2117-24.

23. Mehlhorn U, Allen SJ, Adams DL, Davis KL, Gogola GR, de Vivie ER, et al. Normothermic continuous antegrade blood cardioplegia does not prevent myocardial edema and cardiac dysfunction. Circulation. 1995;92:1940-6.

24. Lieberman R, Padeletti L, Schreuder J, Jackson K, Michelucci A, Colella A, et al. Ventricular pacing lead location alters systemic hemodynamics and left ventricular function in patients with and without reduced ejection fraction. J Am Coll Cardiol. 2006;48:1634-41.

25. Bethea BT, Salazar JD, Grega MA, Doty JR, Fitton TP, Alejo DE, et al. Determining the utility of temporary pacing wires after coronary artery bypass surgery. Ann Thorac Surg. 2005;79:104-7. 